\title{
The Multiple Representations of Complex Digit Movements in Primary Motor Cortex Form the Building Blocks for Complex Grip Types in Capuchin Monkeys
}

\author{
(D)Andrei Mayer, ${ }^{1}$ Mary K.L. Baldwin, ${ }^{2}$ Dylan F. Cooke, ${ }^{3}$ Bruss R. Lima, ${ }^{4}$ Jeffrey Padberg, ${ }^{5}$ Gabriela Lewenfus, ${ }^{4}$ \\ João G. Franca, ${ }^{4}$ and Leah Krubitzer ${ }^{2}$ \\ ${ }^{1}$ Department of Physiological Science, Federal University of Santa Catarina, Florianopolis, SC 88040-900, Brazil, ${ }^{2}$ Center for Neuroscience, University of \\ California Davis, Davis, California 95616, ${ }^{3}$ Department of Biomedical Physiology and Kinesiology, Simon Fraser University, Burnaby, British Columbia V5A \\ 1S6, Canada, ${ }^{4}$ Carlos Chagas Filho Institute of Biophysics, Federal University of Rio de Janeiro, Rio de Janeiro, RJ 21941-902, Brazil, and ${ }^{5}$ Department of \\ Biology, University of Central Arkansas, Conway, Arkansas 72035
}

In the present study, we investigated motor cortex (M1) and a small portion of premotor and parietal cortex using intracortical microstimulation in anesthetized capuchin monkeys. Capuchins are the only New World monkeys that have evolved an opposable thumb and use tools in the wild. Like most Old World monkeys and humans, capuchin monkeys have highly dexterous hands. We surveyed a large extent of M1 and found that $\sim 22 \%$ of all evoked movements in M1 involved the digits, and the majority of these consisted of finger flexions and extensions. Different subtypes of movements could be identified, including opposable movements of digits 1 and 2 (D1 and D2). Interestingly, the pattern of such movements varied between animals. In one case, movements involved the adduction of the medial surface of D1 toward the lateral surface of D2, whereas in the other case, the tips of D1 and D2 came in contact. Unlike other primates examined, we also found extensive representations of the prehensile foot and tail. We propose that the manual behavioral repertoire of capuchin monkeys, which includes the use of tools in the wild, is well represented within the motor cortex in the form of muscle synergies between different body parts that compose these larger, complex behaviors.

Key words: finger; grasping; motor cortex; primate

Significance Statement

The ability to use tools is a milestone in human evolution. Capuchin monkeys are one of the few non-human primates that use tools in the wild. The present study is the first detailed exploration of the motor cortex of these primates using long-train intracortical microstimulation. Within primary motor cortex, we evoked finger movements involving flexions and extensions of multiple digits, or of the first and second digits alone. Interestingly, flexion of tail and toes could also be evoked. Together, these results suggest that the functional organization of the motor cortex represents not just muscles of the body, but muscle synergies that form the building blocks of the complex behavioral repertoire of these animals.

\section{Introduction}

Until relatively recently, the extensive and pervasive use of tools was considered a unique behavior of humans, and one of the

Received March 8, 2019; revised May 17, 2019; accepted June 12, 2019.

Author contributions: A.M., D.F.C., B.R.L., J.P., J.G.F., and L.K. performed experiments; A.M., M.K.L.B., G.L., and J.G.F. analyzed data; A.M. wrote the first draft of the paper; A.M., M.K.L.B., D.F.C., J.P., J.G.F., and L.K. edited the paper; A.M. and L.K. wrote the paper; J.G.F. and L.K. designed research.

This work was supported by National Institute of Health (FIRCA (Fogarty International Research Collaboration Award), Effects of reversible deactivation of posterior parietal cortex in New World (ebus monkeys), National Institutes of Health R03 Grant TW008928, and Conselho Nacional de Desenvolvimento Científico e Tecnológico Grant 402143/2012-4.

The authors declare no competing financial interests.

Correspondence should be addressed to Leah Krubitzer at lakrubitzer@ucdavis.edu. major foundations of our culture. However, stone tool use has now been documented in macaque monkeys in Thailand (Gumert et al., 2009; Gumert and Malaivijitnond, 2013), and in capuchin monkeys in Brazil (e.g., Fragaszy et al., 2004; Visalberghi et al., 2009). This lithic percussive behavior requires the implicit knowledge of object features, such as size, shape, and density, and how objects can be manipulated to alter or transform other objects. In capuchins, these abilities and other behaviors that require complex digit movements co-evolved with an elaboration of motor areas, such as primary motor cortex (M1), sup- 

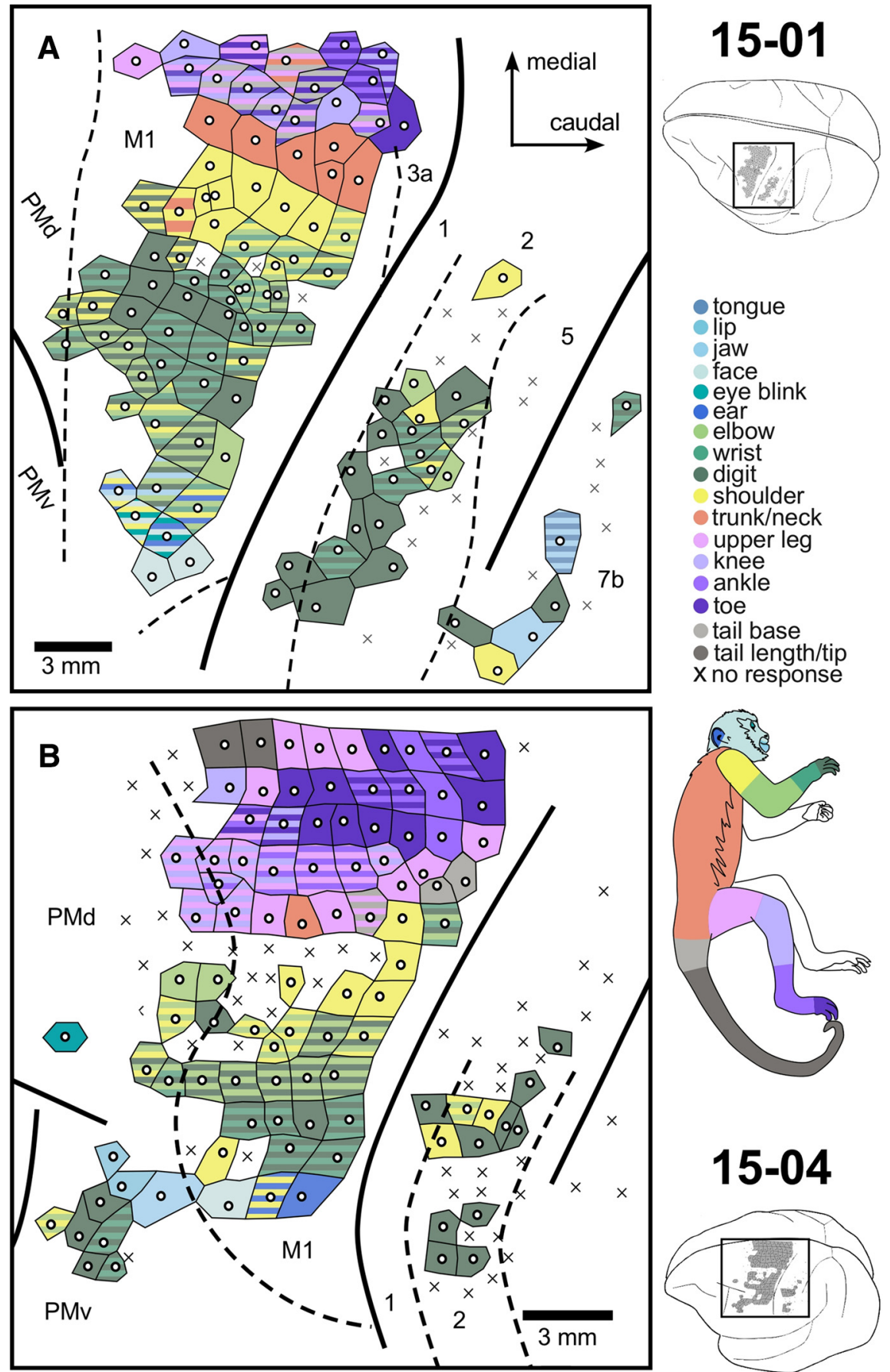

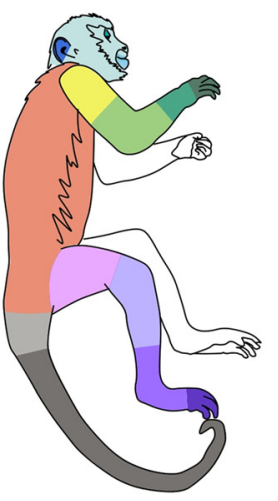

15-04

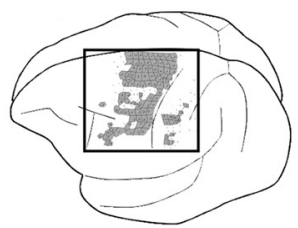

Figure 1. LT-ICMS map for Cases 15-01 and 15-04. Enlarged representation of the cortical surface region mapped in the frontal and parietal cortex of the left hemisphere of Cases 15-01 $(\boldsymbol{A})$ and 15-04 (B), corresponding to the black rectangles in the brain drawings (top and bottom right corners). Movements here were categorized according to the body part(s) that moved during LT-ICMS. White dots represent the electrode penetration site. Tiles surrounding the white dots represent the body part(s) involved in the evoked movement according to the color code at right. Striped tiles represent movements involving multiple body parts. X's indicate sites where no movement was elicited during LT-ICMS. Dashed lines indicate anatomical borders between cortical areas defined by Nissl-stained sections.

plementary motor area (SMA), ventral premotor cortex (PMv), and dorsal premotor cortex (PMd) (e.g., Dum and Strick, 2005; Dea et al., 2016; Hamadjida et al., 2016; Côté et al., 2017), the addition of parietal areas, such as area 2 and an expanded area 5 (e.g., Padberg et al., 2007; Mayer et al., 2016, 2019), the emergence of specializations in the skeletal morphology of the hand (e.g., Spinozzi et al., 2004, 2007; Aversi-Ferreira et al., 2011), and

\section{5-01}

tongue

lip

- jaw

face

eye blink

ear

elbow

owrist

- digit

shoulder

trunk/neck

opper leg

knee

ankle

- toe

tail base

- tail length/tip

$\mathrm{X}$ no response

corticospinal projections involved in fine control of individual digits (Heffner and Masterton, 1983; Bortoff and Strick, 1993). However, these features of organization have evolved independently from similar alterations in the body and brain of Old World primates, and are not present in other New World monkeys. Thus, while most New World monkeys exclusively perform whole-hand grips during object manipulation, capuchin monkeys can execute up to 16 different types of precision grips (Christel and Fragaszy, 2000; Spinozzi et al., 2004, 2007). Furthermore, capuchin monkeys spontaneously use tools in the wild. They use rocks as hammers and anvils (Fragaszy et al., 2004; Moura and Lee, 2004; Visalberghi et al., 2007), manufacture tools (e.g., by shaping sticks as insect probing tools) (Mannu and Ottoni, 2009), and select the best tool for a specific task based on its physical properties (Visalberghi et al., 2009; Manrique et al., 2011).

Despite their extraordinarily dexterous manual behaviors, the functional organization of their motor cortex has not been explored in detail. In the early 1970s, Asanuma and Rosén (1972) evoked specific finger movements in M1 of capuchins (i.e., extension, flexion, adduction, and abduction) using short-train intracortical microstimulation (ST-ICMS), and recently a large representation of the digits was described by Hamadjida et al. (2016). Subsequent studies focused on the neural response properties of M1 (Rosén and Asanuma, 1972; Conrad et al., 1974; Lucier et al., 1975), its connectivity (Hoover and Strick, 1999; Kelly and Strick, 2003; Dum and Strick, 2005; Dea et al., 2016; Hamadjida et al., 2016), and M1's functional relationship with the premotor cortex (PM) (Ohbayashi et al., 2016; Quessy et al., 2016; Côté et al., 2017). In most of these studies, only a small portion of M1 was explored. Furthermore, except for the study of Asanuma and Rosén (1972), no other study in capuchins has described the details of the evoked movements of different body parts.

In the current study, we use long-train intracortical microstimulation (LT-ICMS) to define the functional organization of motor cortex in capuchins; LT-ICMS evokes relatively complex (i.e., multi-joint and multi-body part) movements, instead of isolated muscle contractions (Graziano et al., 2002). Further, LTICMS has been used as a robust technique in a wide range of mammals to compare organizational features of motor and parietal cortex (for review, see Graziano, 2016; Baldwin et al., 2018). 


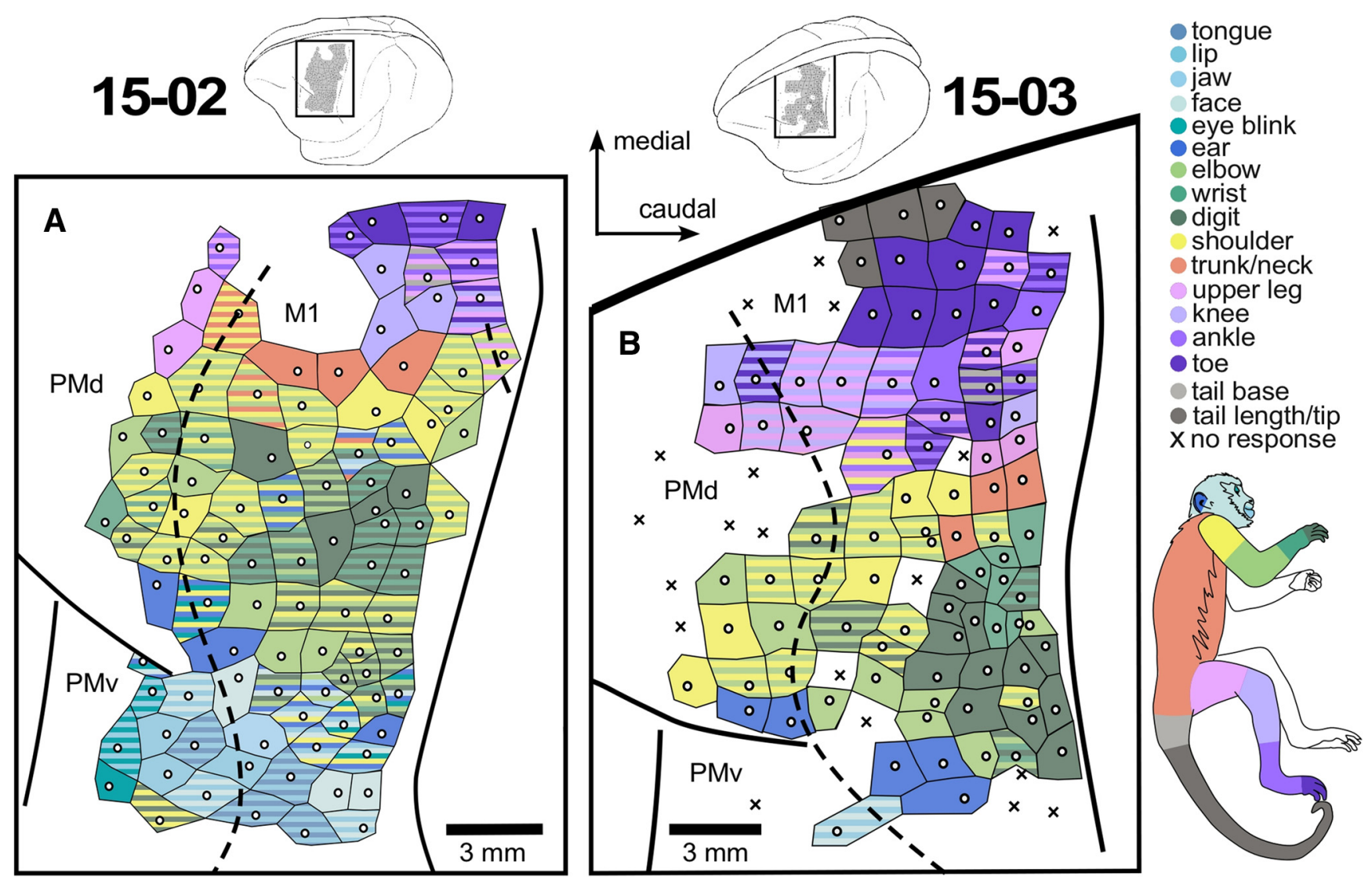

Figure 2. LT-ICMS map for Cases 15-02 and 15-03. Enlarged representation of the cortical surface region mapped in the frontal cortex of the lefthemisphere of Cases 15-02 (A) and 15-03 (B), corresponding to the black rectangles in the brain drawings above each of the enlarged maps. Movements here were categorized according to the body part(s) that moved during LT-ICMS. White dots represent the electrode penetration site. Tiles surrounding the white dots represent the body part(s) involved in the evoked movement according to the color code at right. Striped tiles represent movements involving multiple body parts. X's indicate sites where no movement was elicited during LT-ICMS. Dashed lines indicate anatomical borders between cortical areas defined by Niss--stained sections.

In our previous studies of parietal cortex, we proposed that there are inescapable outcomes of cortical organization that emerge due to homologous developmental programs that unravel in the context of specialized hand morphology and the use of a limb (Padberg et al., 2007). The goal of the present investigation was to determine if this was also true for motor cortex organization. Here we explore how digit and hand movements are represented in motor cortex in capuchin monkeys, and how these movement representations of the digits compare with those of other primates. We hypothesize that motor cortex organization is not a manifestation of tool use per se, but rather co-evolved with modifications to the hand and its biomechanics, which allows for multiple complex grip types, and is built during development based on commonly used muscle synergies.

\section{Materials and Methods}

Experimental design and statistical analysis. The organization of motor and parietal cortex was examined using LT-ICMS in 4 adult (age unknown), anesthetized capuchin monkeys

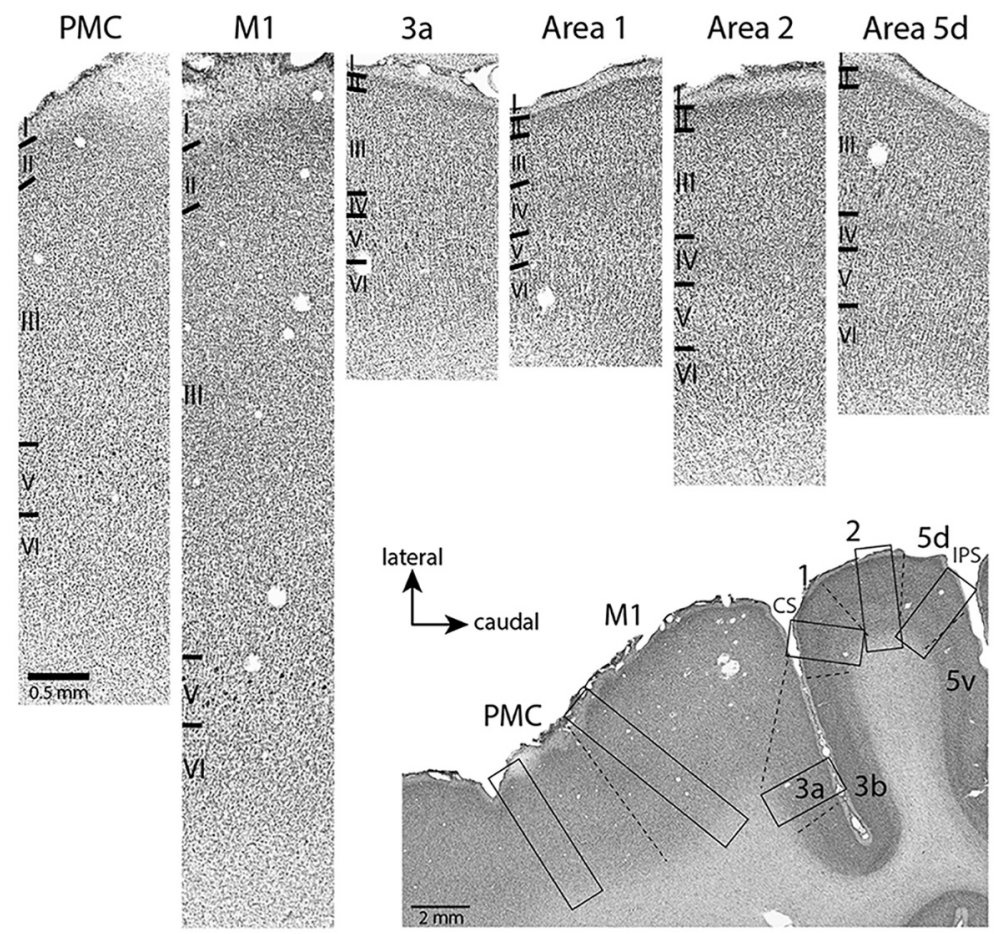

Figure 3. Photomicrographs showing the architecture of frontal and parietal areas revealed by Nissl staining. Photomicrographs at the top were all taken from the same horizontal section shown in right bottom corner. Black rectangles correspond to cortical regions enlarged above. Dashed lines indicate anatomical borders. The scale bar in the top left image (PMC) is the same for all top images. PMC, Premotor cortex. 


\section{A Arm elevation + Forearm supination + Elbow and digit flexion}

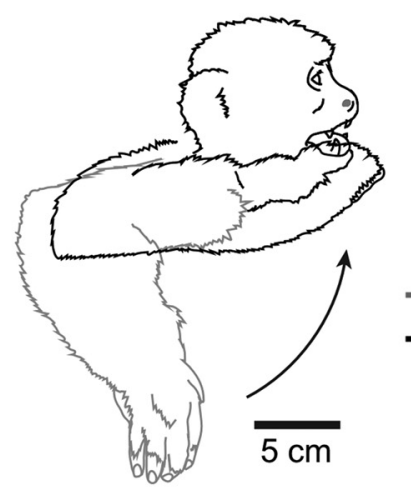

\section{B Ear and lip retraction + eye blink + face muscles contraction + mouth open}

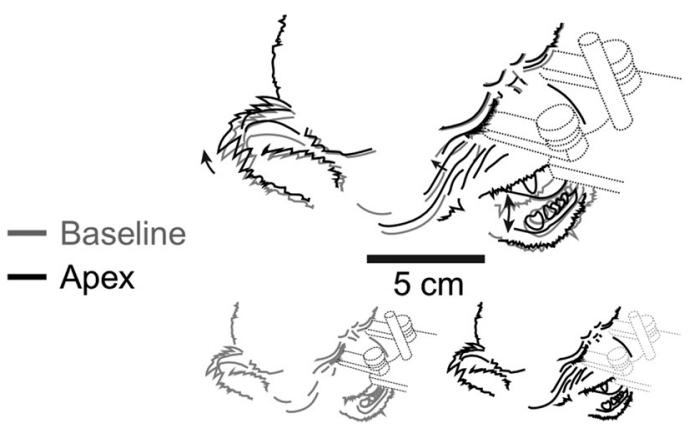

\section{Arm elevation + Elbow flexion
+ digit extension \\ C Arm elevation + Elbow flexion
+ digit extension}

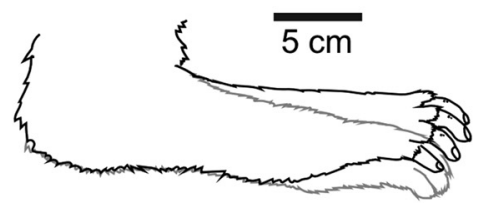
D Proximal hindlimb and ankle flexion + arm forward

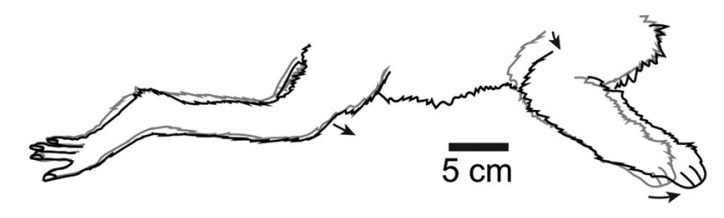

Figure 4. Examples of multi-joint/multi-body part movements evoked after LT-ICMS in M1.A, Movement evoked from site 98 of Case 15-01 (see Figure 1-1, available at https://doi.org/10.1523/ JNEUROSCI.0556-19.2019.f1-1). This movement involved an elevation of the entire arm plus forearm supination and elbow flexion, which brought the hand to the mouth. $\boldsymbol{B}$, Movement evoked from site 73 of Case 15-02 (see Figure 2-1, available at https://doi.org/10.1523/JNEUROSCI.0556-19.2019.f2-1). Stimulation at this site evoked movements of the face (e.g., mouth closing; cheeks elevating) accompanied by shoulder elevation. C, Movement evoked from site 16 of Case 15-02 (see Figure 2-1, available at https://doi.org/10.1523/JNEUR0SCI.0556-19.2019.f2-1) involved an arm elevation, elbow flexion, and digit extension. D, Movements evoked from site 68 of (ase 15-01 (see Figure 1-1, available at https://doi.org/10.1523/JNEUROSCI.0556-19.2019.f1-1) involved both the forelimb and hindlimb. Drawings were obtained from video frames taken from two different moments: (1) before ICMS (i.e., body in the starting position; gray lines); and (2) at the apex of the movement during ICMS (black lines).

(Sapajus spp. formerly Cebus spp.) (Martins et al., 2015) of both sexes (3 males and 1 female) weighing between 1.9 and $4.6 \mathrm{~kg}$. Movements evoked by LT-ICMS were digitally recorded from two separate angles (Sanyo Xacti VPC-HD2000A, $1920 \times 1080$ resolution, 60 frames/s) and analyzed offline (see Movement analysis). Each experiment revealed variations in the movement maps generated, but conclusions were drawn based on similarities between individual animals. Raw data (uninterpreted) for each experiment are presented in Fig. 1-1 (available at https://doi.org/10.1523/JNEUROSCI.0556-19.2019.f1-1), Fig. 1-2 (available at https://doi.org/10.1523/JNEUROSCI.0556-19.2019.f12), Fig. 2-1 (available at https://doi.org/10.1523/JNEUROSCI.055619.2019.f2-1), and Fig. 2-2 (available at https://doi.org/10.1523/ JNEUROSCI.0556-19.2019.f2-2). We only used statistical tests to analyze differences in the threshold values between cortical regions (i.e., premotor vs motor vs parietal cortices). For that, we used a standard two-tailed unequal variance $t$ test in Microsoft Excel.

Surgical procedures. Animals were obtained from the animal facility of the Institute of Biophysics Carlos Chagas Filho, under the license of the Brazilian Institute of the Environment and Renewable Natural Resources. All experimental procedures were approved by the local animal care and use committee (CEUA-CCS/UFRJ, protocol \#IBCCF-123) and are in accordance with the Brazilian Law and National Institutes of Health guidelines.

Animals were initially anesthetized with an intramuscular (IM) injection of ketamine $(20-50 \mathrm{mg} / \mathrm{kg})$. Once anesthetized, atropine $(0.05-0.12$ $\mathrm{mg} / \mathrm{kg} ; \mathrm{IM})$, diazepam $(0.5-1.0 \mathrm{mg} / \mathrm{kg} ; \mathrm{IM})$, and dexamethasone $(0.4-$ $1.0 \mathrm{mg} / \mathrm{kg}$; IM) were administered. Animals were then intubated, cannulated, and catheterized. Surgical levels of anesthesia were maintained with supplemental doses of ketamine (1-25 mg/kg) and xylazine $(0.01-$
$0.03 \mathrm{mg} / \mathrm{kg}$ ) delivered intramuscularly, as well as a continuous infusion of ketamine administered intravenously (1-35 mg/kg/h). In 3 animals, the inhalant anesthetic halothane $(0.25 \%-2 \%)$ was used in conjunction with the ketamine infusion. Heart rate, respiration rate, body temperature, muscle tone, and reflexes were monitored throughout the experiment to assure a constant level of anesthesia.

After animals were anesthetized, subcutaneous injections of lidocaine (2\%) were placed at the ears, and topical lidocaine was applied to the external ear canals. Animals were then placed in a stereotax and positioned such that their upper trunk and forelimbs were unobstructed. Ophthalmic ointment was placed in the eyes to prevent drying. Subcutaneous injections of lidocaine $(2 \%)$ were made at the midline of the scalp before making a surgical incision to expose the skull. The scalp was cut, the temporal muscles were retracted, and a craniotomy was made to expose portions of frontal and parietal cortex. Once the dura was removed, dimethylpolysiloxane was placed over the cortex to prevent desiccation, and the cortical surface was photographed and printed to directly relate stimulation site locations with cortical vascular patterns and sulcal landmarks.

ICMS motor mapping. Stimulation was generated with a Grass S88 stimulator (RRID:SCR_016192) and two SIU6 stimulus isolation units and delivered using a low impedance $(0.1 \mathrm{M} \Omega)$ microelectrode. Amplitude, duration, and frequency of stimulation were measured by the voltage drop across a $10-\mathrm{k} \Omega$ resistor in series with the return lead of the stimulation isolation units. An LED light source positioned in the video frame and loudspeaker were connected to the stimulator sync output to provide stimulation train timing in visual and auditory form. Electrical stimulation consisted of $500 \mathrm{~ms}$ trains of biphasic square wave pulses (two $0.2 \mathrm{~ms}$ pulses) delivered at $200 \mathrm{~Hz}$. 
The stimulation electrode was lowered into the cortex to a depth of $1500 \mu \mathrm{m}$ (to layers V and VI) using a micromanipulator. To stimulate cortex within the sulci, the electrode was advanced parallel to the pial surface, and stimulation sites were tested every $500 \mu \mathrm{m}$ down to a maximum depth of $8 \mathrm{~mm}$. We began exploring different cortical fields using stimulation pulses with an amplitude of $50 \mu \mathrm{A}$. However, if this current was not strong enough to elicit a movement, we increased the amplitude. If no movement was detected for amplitudes up to $300 \mu \mathrm{A}$ in the frontal cortex, or up to $600 \mu \mathrm{A}$ in the parietal cortex, the site was labeled as "no response." Fiducial probes (fluorescent dyes) were placed at different locations at the end of the experiment to aid in the alignment of functional and histological data (see below).

Histological procedures. When ICMS mapping was complete, animals were given a lethal dose of sodium pentobarbital, and perfused transcardially with saline, followed by either $2 \%$ or $4 \%$ paraformaldehyde (PFA), then $2 \%$ or $4 \%$ PFA with $10 \%$ sucrose added, $\mathrm{pH} 7.3$. The brains were removed, postfixed with $4 \%$ PFA for 3-36 h, and then placed in a $30 \%$ sucrose solution for $48-60 \mathrm{~h}$.

The brain was blocked to include only the region of cortex posterior to the arcuate sulcus, anterior to the lunate sulcus, and dorsal to the posterior inferior temporal sulcus. This block was sectioned horizontally while a camera (Nikon DSLR 5200) mounted over the brain collected block-face images of each section. The tissue was cut at a thickness of $60 \mu \mathrm{m}$ using a freezing microtome and processed in four series. One series of sections was processed for Nissl and used for identifying borders of cortical areas. The remaining series were saved for another study.

The boundaries of areas $3 \mathrm{a}, 3 \mathrm{~b}, 1,2,5 \mathrm{v}$, and $5 \mathrm{~d}$ were identified on Nissl-stained sections using the architectonic criteria established by Mayer et al. (2016) in capuchin monkeys. The rostral boundary of area M1 was determined using the same criteria used for macaque monkeys (Vogt and Vogt, 1919; Jones et al., 1978; Baldwin et al., 2018). We did not distinguish between subdivisions of PM (e.g., F2, F5, and F4) (Luppino and Rizzolatti, 2000) but instead only defined the larger subdivisions of PMv, located rostral to $\mathrm{F} 1$ and ventral to the superior arcuate sulcus, and PMd, located rostral to F1 and dorsal to the superior arcuate sulcus.

Physiological and anatomical reconstruction.

Block-face images of all cortical sections were processed in Photoshop (Adobe) (see Baldwin et al., 2018). Cortical borders identified in Nisslstained sections were superimposed onto the corresponding blockface images, as were the locations of fiducial probes. These images were then imported into Fiji processing package (Schindelin et al., 2012), and a 3D reconstruction was made using the "3D view" plug-in. The $3 \mathrm{D}$ reconstructions were then aligned to images of the surface of the brain using fiducial probes, penetration sites, and local landmarks, including sulci and gyri. This process allowed us to accurately align our microstimulation sites with architectonically defined cortical field boundaries.

Movement analysis. During the experiments, all movements were characterized by 2 observers and digitally recorded. These movements were later confirmed and analyzed offline. Movements were characterized by
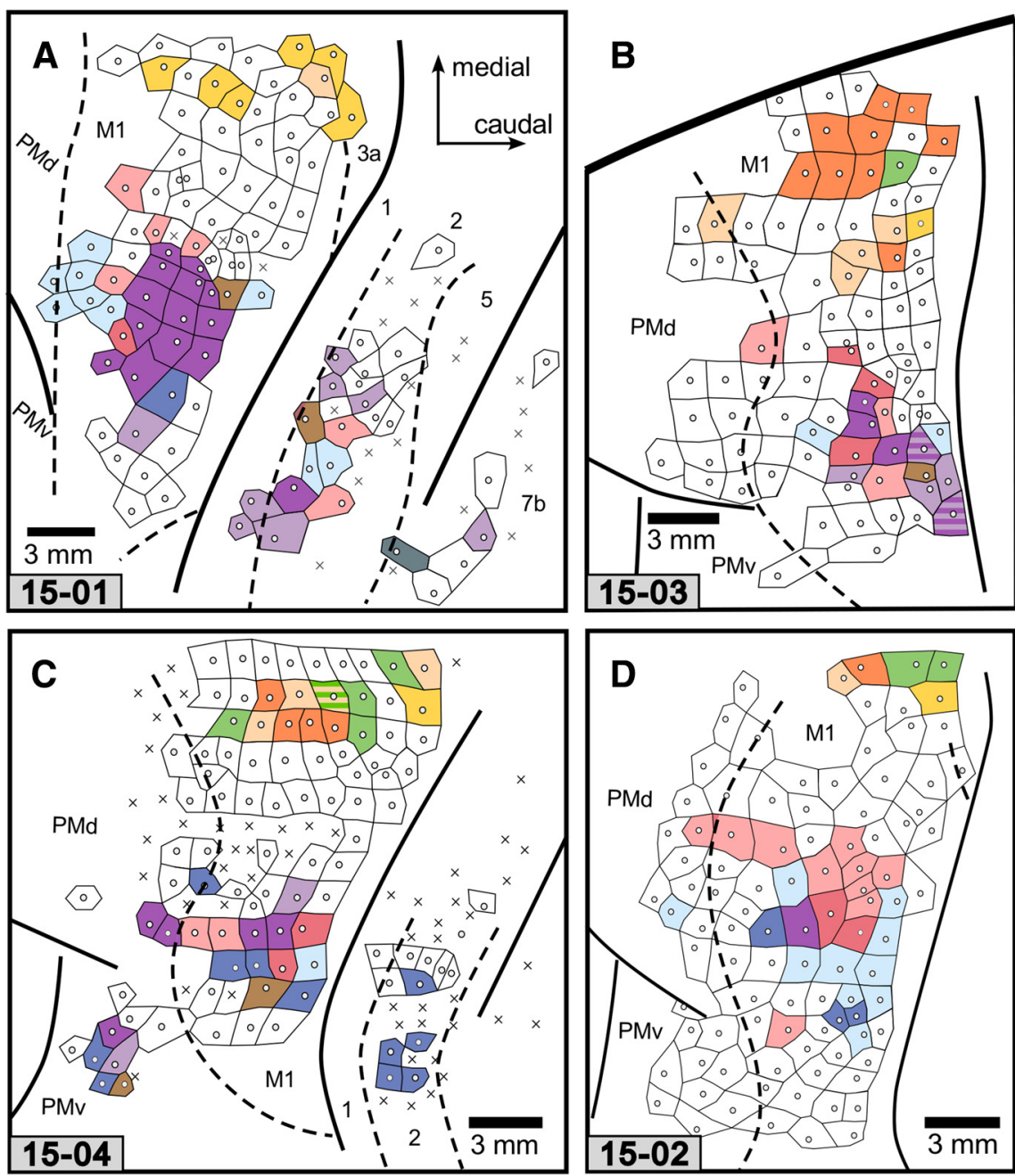

Distal portion of D1 touches the middle or distal portion of any digit

Flexion of D1 and/or D2

Flexion of all digits towards the palm

Other movements involving flexion of more than 2 digits or D3 or D4 or D5 Extension of more than 2 digits or D3 or D4 or D5

Mix of flex and ext of only D1 and D2

Flex of all toes towards the foot sole

Flexion of more than 2 toes or T3 or T4 or T5

Flexion of $\mathrm{T} 1$ and/or T2

Extension of more than 2 toes or T3 or T4 or T5

Figure 5. Movement map for digits and toes. A, Case 15-01. B, Case 15-03. C, Case 15-04. D, Case 15-02. Some LT-ICMS sites that elicited digit flexion fell into two major categories: one in which digit 1 was opposed to one or more of digits $2-5$; and one in which one or more digits flexed toward the palm. Interestingly, these categories were subsets of digit movements used by capuchin monkeys in the wild (Costello and Fragazy, 1988; Spinozzi et al., 2004). The color code of this map is indicated at the bottom. Other conventions are the same as in Figure 3.

the joint and body part moving, such as the wrist or tongue. When multiple joints or body parts moved together, these movements were labeled as "complex" movements. Because we were interested in specific digit movements associated with grasping behaviors, we defined digit movements based on whether the movements were due to joint flexion, extension, abduction, or adduction.

Representative movements were illustrated from frames captured just before stimulation initiation (baseline), and at the peak of the movement amplitude (apex). These frames were imported into Adobe Illustrator where the outline of the body part(s) involved in the movement was traced. We did not monitor eye movements.

The total area of M1 from which digit movements could be evoked was measured. First, a Voronoi tessellation of the motor map of each case was created based on the location of ICMS sites to have a more precise and 
A

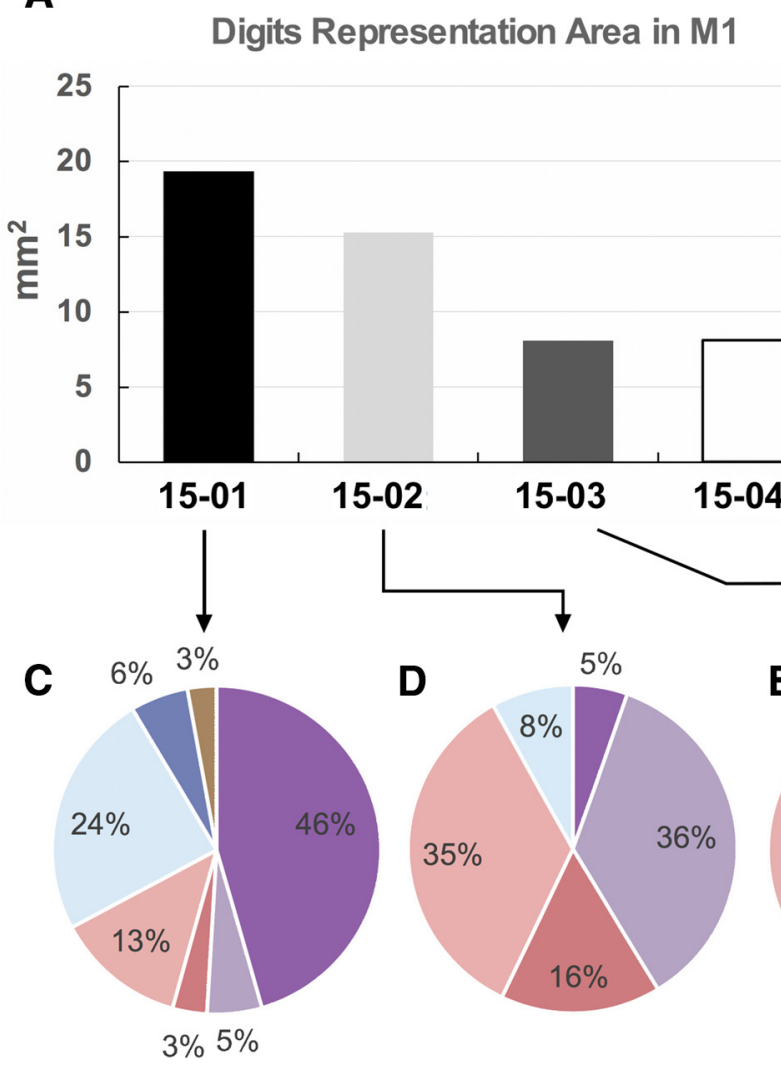

B

Distal portion of D1 touches the middle or distal portion of any digit

Flexion of D1 and/or D2

Flexion of all digits towards the palm

Other movements involving flexion of more than 2 digits or D3 or D4 or D5

\section{Extension of more than 2 digits or D3 or D4 or D5}

Extension of D1 and/or D2

Mix of flex and ext of only D1 and D2

Figure 6. Quantification of evoked digit movement types in the hand representation of M1. $\boldsymbol{A}$, Total area of hand representation within M1. Only sites that evoked digits movements were considered (Fig. 5). B, Color code of the pie charts presented in C-F. C-F, Proportion of different types of digits movements within the hand representation of each case. In all cases, most evoked digit movements in $\mathrm{M} 1$ involved D1 touching any other finger or the flexion of D1 and/or D2 alone.

reliable border between these sites (for details, see Baldwin et al., 2018). Then the area of all sites in the motor map at which digit movements were evoked was also measured using Adobe Illustrator script. The area for which a specific type of digit movement could be evoked was also measured. This was done by taking the area within M1 from which the specific digit movement could be evoked and dividing it by the total area of M1 for which any digit movement could be evoked. Specific digit movements were classified into seven groups and included the following: (1) distal portion of D1 touches the middle or distal portion of any digit; (2) flexion of D1 and/or D2; (3) flexion of all digits; (4) other movements involving flexion of $>2$ digits or D3 or D4 or D5; (5) extension of $>2$ digits or D3 or D4 or D5; (6) extension of D1 and/or D2; and (7) mix of flexion and extension of only D1 and D2. Thus, it was possible to calculate the proportion of $\mathrm{M} 1$ within the digit region that evoked each type of digit movement (see Fig. 6). When an ICMS site could be categorized as two different types of movements (see, e.g., Fig. $5 B$, purple/lavender striped sites), half of the area of these sites was considered as part of one category and the other half was considered as part of the other category.

Current threshold. The threshold was determined for each site by lowering the current amplitude by half (e.g., from $100 \mu \mathrm{A}$ to $50 \mu \mathrm{A}$, and then to $25 \mu \mathrm{A}$ ) until no movement could be evoked. The halfway point between the current value that resulted in no movement and the previous current applied in which the movement could be evoked was defined as the threshold. To confirm the stability of anesthesia, and therefore our ability to consistently evoke movements throughout the experiment, we periodically returned to stimulation sites to retest threshold values. Differences in threshold values across cortical areas and regions were tested using a standard two-tailed unequal variance $t$ test in Microsoft Excel.

\section{Results}

In the present study, 483 sites within frontal and parietal cortex were stimulated using LT-ICMS in 4 capuchin monkeys ( 3 males and 1 female). Of these, movements could be evoked from 298 of sites within M1, 55 within parietal areas, and 38 within premotor areas. Movement maps for our 4 cases are illustrated in Figures 1 and 2, and descriptions of the movements at each microstimulation site that were used to generate these maps are presented in Fig. 1-1 (available at https://doi.org/10.1523/JNEUROSCI.055619.2019.f1-1), Fig. 1-2 (available at https://doi.org/10.1523/ JNEUROSCI.0556-19.2019.f1-2), Fig. 2-1 (available at https:// doi.org/10.1523/JNEUROSCI.0556-19.2019.f2-1), and Fig. 2-2 (available at https://doi.org/10.1523/JNEUROSCI.0556-19.2019. f2-2). We first describe the architectonic appearance of the cortical field from which movements were evoked, including M1, PM, areas 3a, 1, 2, and 5 (Fig. 3), and the general movement map organization in motor and parietal cortex (Figs. 1, 2 ). We next provide details on the types of hand and foot movements that were evoked in motor and parietal cortex, followed by movement thresholds for the different fields.

\section{Movement representations in motor and parietal cortex and their relation to architectonically determined cortical field boundaries}

Stimulation sites were directly related to cortical field boundaries using Nissl-stained horizontal sections. Parietal areas were identified according to the criteria previously described by Mayer et al. (2016) for capuchin monkeys and by Baldwin et al. (2018) for macaque monkeys. Briefly, M1 was characterized by a poorly developed layer 4 , and the presence of large pyramidal cells distributed throughout layer 5 (Fig. 3); whereas in the premotor areas (PMd and PMv), the cells in layer 5 were smaller and sparser (Fig. 3). Area 3 a was characterized by a well-developed layer 3 and the pres- 
ence of sparsely distributed large pyramidal cells in layer 5 . Area 1 had a striated appearance with moderate staining of layer 4 , and area 2 exhibited much less densely staining and densely packed layers 4 and 6. Area 5d had well-developed layers 3 and 5 (Fig. 3).

Across all cortical areas from which movements could be evoked, there was a general topographic organization that has been described in other primates and other mammals (for review, see Baldwin et al., 2018). Movements could be elicited from frontal and parietal cortical areas. The majority $(76 \%)$ of microsimulation sites from which movements could be evoked were located within the M1 (Figs. $1,2)$, in which the full contralateral extent of the body was represented, including the face, forelimb, hindlimb, trunk, and tail. A rough somatotopic organization could be identified, with hindlimb and tail represented medially and forelimb and face laterally.

In all cases, forelimb movements could also be evoked from sites located rostral to the anterior border of M1 (Figs. 1, 2), in PM. In 3 cases, movements of the face, trunk, and portions of the hindlimb were also evoked from PM (Figs. $1 B, 2$ ). In 2 cases, forelimb movements could be evoked from anterior parietal areas 1 and 2 (Fig. 1); and in 1 case, face movements were evoked from the posterior parietal area $7 \mathrm{~b}$ (Fig. $1 A$ ).

In all cases, at least one of two types of tail movement could be evoked from M1: (1) contraction of its base, with no particular direction; and (2) flexion of its distal portion. The former type was evoked in all cases and, except for two ICMS sites in Case 15-04 (Fig. 1B), it was always accompanied by the movement of other body parts, especially the hindlimb (Figs. 1, 2, striped light gray with shades of purple). On the other hand, flexion of the distal portion of the tail was always isolated from movements of other body parts, although the number of sites evoking this type of movement was relatively small (two sites in Case 15-04 and four in Case 15-03; Fig. $1 B$ and Fig. $2 B$, respectively).

Most of the evoked movements involved multiple joints and/or multiple body parts. Some examples of these movements are illustrated in Figure 4. Some investigators have classified these as "ethologically relevant behaviors," such as "hand to mouth," "face defense," and "reach" (e.g., Stepniewska et al., 2005). Rather than use this terminology, we decided to describe evoked movements by reporting co-displacement patterns of different joints, which allows for more precise descriptions of synergies (see Fig. 1-1, available at https://doi.org/10.1523/JNEUROSCI.0556-19.2019.f1-1; Fig. 1-2, available at https://doi.org/10.1523/JNEUROSCI.0556-19.2019. f1-2; Fig. 2-1, available at https://doi.org/10.1523/JNEURO-

\section{Dorsal view}

\section{Ventral view}

\section{FLEX OF ALL DIGITS}

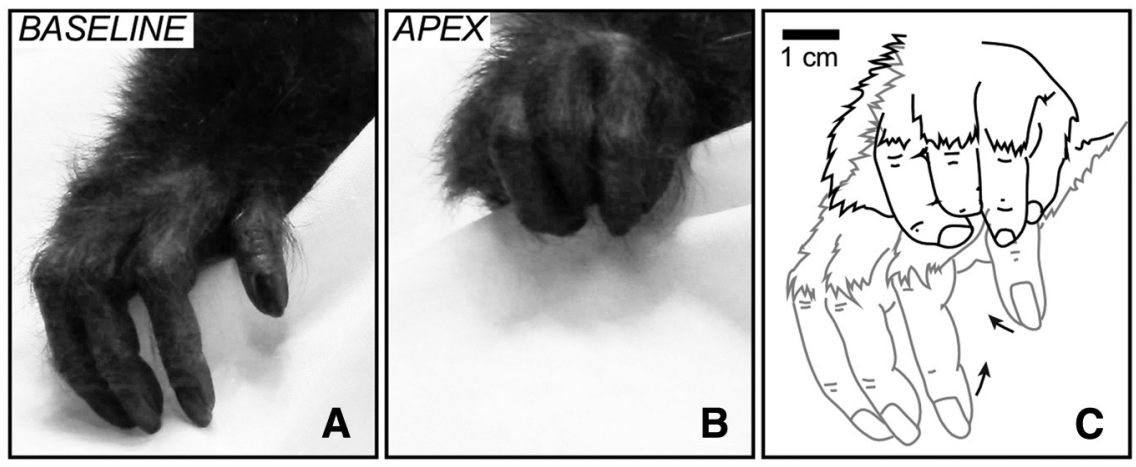

\section{FLEX OF ALL TOES}
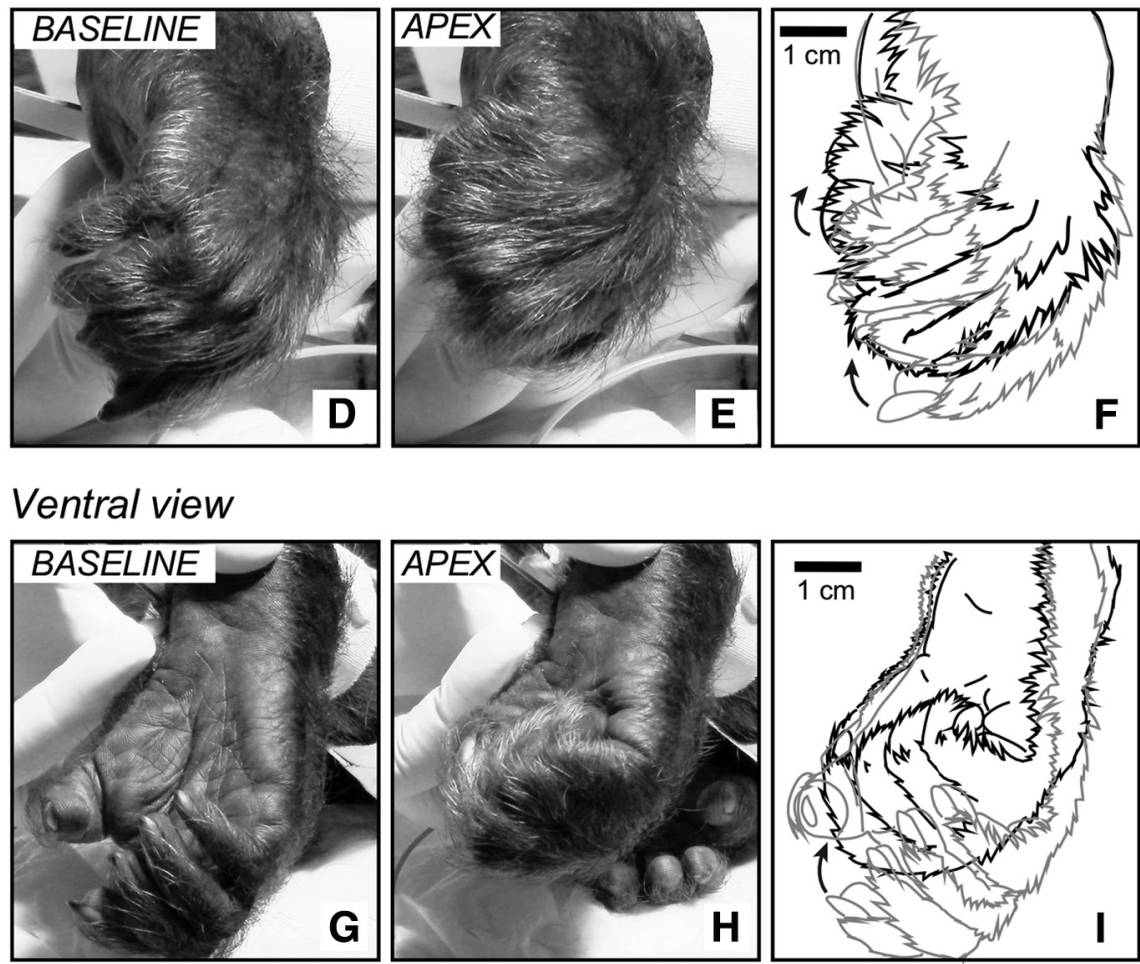

Figure 7. Examples of evoked hand and foot movements. A-C, Evoked hand movements in which all digits flex toward the palm. In this particular case (Case 15-03), the animal was missing D5 in the right hand. This movement was evoked at site 11 (see Figure 2-2, available at https://doi.org/10.1523/JNEUROSCI.0556-19.2019.f2-2). Dorsal (D-F) and ventral (G-I) views of the foot in which all toes flex toward the sole of the foot; site 115 of Case 15-03 (see Figure 2-2, available at https://doi.org/10.1523/ JNEUROSCI.0556-19.2019.f2-2). C, F, I, Tracing from video frames showing the starting position (light gray line) and the apex (black line) of each movement.

SCI.0556-19.2019.f2-1; Fig. 2-2, available at https://doi.org/10.1523/ JNEUROSCI.0556-19.2019.f2-2; and Fig. 4). We focus on characterizing movements of the digits and toes.

\section{Characterization of hand and foot movements}

From the 391 ICMS sites that evoked movement in the frontal and parietal cortex of all cases, finger movements were evoked from $22 \%$ of sites, and toe movements were evoked from $10 \%$ of sites. Figure 5 illustrates a map of these movements, classified according to the fingers or toes that moved and how they moved. No clear topographic organization could be observed 
MOSTLY D1
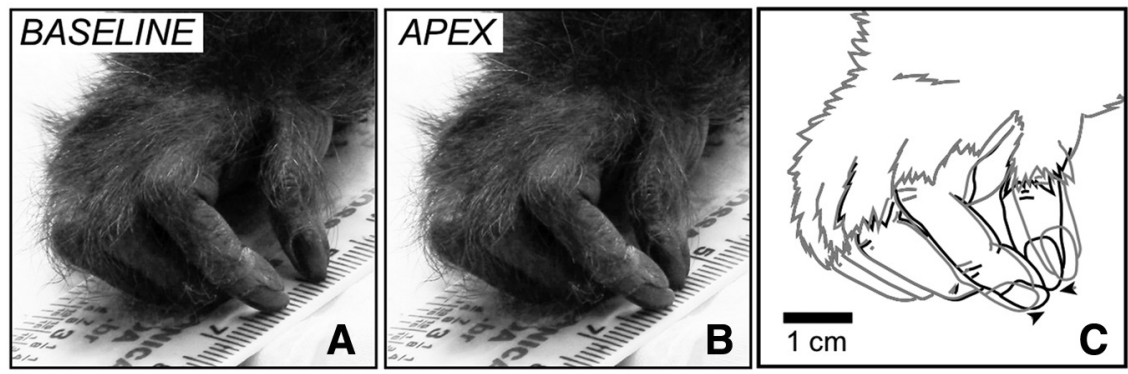

MOSTLY D1 AND D2
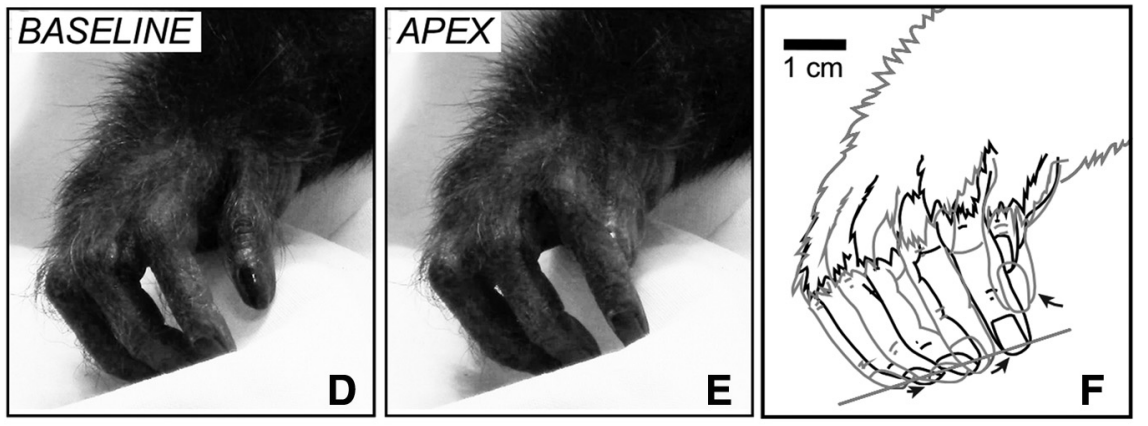

\section{COMPLEX DIGIT MOVEMENTS}
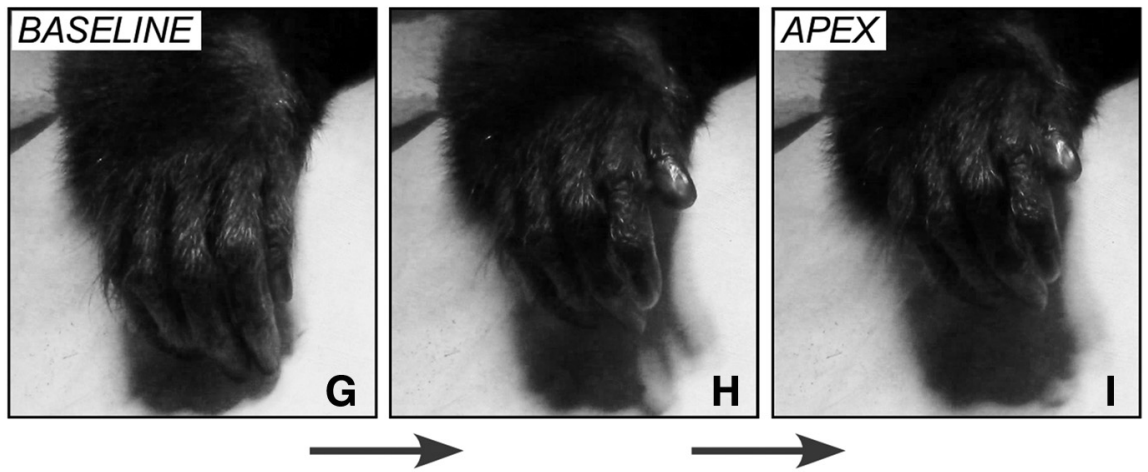

\section{PHASE 1}

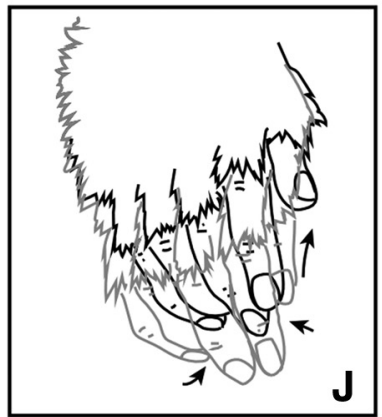

PHASE 2

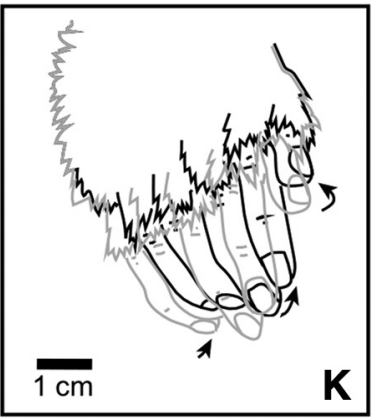

Figure 8. Examples of evoked movements involving D1 and D2. $A-C$, Evoked movements in which the tips of D1 and D2 oppose each other ( $\boldsymbol{B} ; \mathrm{D} 2$ also moves but with a smaller amplitude; see ruler under the fingers), evoked by LT-ICMS at site 98 of Case 15-03 (see Figure 2-2, available at https://doi.org/10.1523/JNEUROSCI.0556-19.2019.f2-2). D-F, A movement in which D2 is adducted, while $D 1$ abducts and extends and its tip touches the second phalange of $D 2$ (other digits also move). This movement was observed in site 9 of Case 15-03 (see Figure 2-2, available at https://doi.org/10.1523/JNEUROSCI.0556-19.2019.f2-2). The tips of D2- 4 are occluded in $\boldsymbol{D}$ and $\boldsymbol{E}$ but were reconstructed in the drawings shown in $\boldsymbol{F}$. $\mathbf{G} \boldsymbol{K}$, Relatively complex movement of the digits in which a D1-D2 opposition first opens $(\boldsymbol{H})$ and then closes so that the distal phalange of D1 touches the proximal and middle phalanges of D2 (I); evoked by LT-ICMS at site 149 of Case 15-04 (see Figure 1-2, available at https://doi.org/10.1523/JNEUROSCI.055619.2019.f1-2).J, Illustrates the first phase of the "complex" movement. Light gray line indicates the starting position (baseline, G). Black line indicates the end of the first phase $(\boldsymbol{H}) . \boldsymbol{K}$, Illustrates the second phase of the movement. Other conventions are the same as in Figure 6. when comparing ICMS sites that evoked finger flexions versus finger extensions. Rather, the maps of these movements were organized in a mosaic fashion. For instance, in 1 case (Fig. $5 \mathrm{~A}$ ), a "finger extension zone" (dark and light blue tiles) in M1 could be identified rostral to a "finger flexion zone" (purple/red tiles); while in another case (Fig. 5D), finger extension was located lateral to finger flexion. Figure 5 illustrates the details of how these movement representations were clustered in M1.

Figure 6 shows the total cortical area occupied by the finger representation within M1 (ranging from $8.1 \mathrm{~mm}^{2}$ in Cases 15-03 and 15-04 to $19.3 \mathrm{~mm}^{2}$ in Case 15-01) as well as the proportion of each digit movement type. In all cases, most evoked digits movements in M1 involved D1 touching any other finger or the flexion of D1 and/or D2 alone, ranging from $33 \%$ to $51 \%$ of the total cortical hand/digit area within M1 (calculated by the sum of the purple parts of the pie charts in Fig. $6 C-F$ ). In all cases, the cortical area within M1 dedicated to only digit flexions (ranging from $63 \%$ to $92 \%$; sum of the purple and red parts in the pie charts of Fig. 6) was at least twice the size of the area that evoked only digit extensions $(8 \%-30 \%$; sum of the blue parts in the pie charts).

As expected, toe movements were evoked along the most medial aspect of each ICMS motor map. The number of ICMS sites from which toe flexions compared with toe extensions could be evoked, as well as their location in relation to each other, was highly variable (Fig. 5), although this could be a result of the small number of toe sites explored. Overall, most evoked toe movements were related to the flexion of more than two toes (usually all toes flexed together; Fig. $7 D-I$ ), followed by the extension of more than two toes (Fig. $5 B-D$ ). However, in 1 case (Fig. 5A), most evoked foot movements were a flexion of only $\mathrm{T} 1$.

One of the major findings of the current study was the observation of two specific types of hand movements: (1) when digit 1 touched the middle or distal portions of any of the other four fingers (Figs. $8,9)$; and (2) when all fingers flexed toward the palm (Fig. $7 A-C$ ). A similar movement type was observed for the foot where all toes flexed toward the plantar surface of the foot (Fig. 7D-I). Among all sites within the motor cortex in which D1 touched any other finger, the most common type of movement was the opposition between D1 and D2 (73\% of all 24 
sites of all cases; Figs. 6, 8, 9G-I). This type of evoked movement of the digits varied between animals. In 1 case, this movement was achieved mostly by the adduction of D1 toward D2 such that the ulnar surface of D1 touched the radial surface of D2 (Fig. 9G-I). In another case, the volar aspect of D2 was mostly touched by the tip of D1 (Fig. $8 A-F$ ). Other variations consisted of the opposition between D1 and D2+D3 (18\%; Fig. 9A-C), and between the tip of D1 and other fingers (9\%; Fig. $9 D-F)$. However, it is important to note that this quantification of different types of digit movements does not have any statistical validity, which was not possible due to the low number of sites that could be categorized (varying between 1 and 13 among cases; Fig. $5 D$ and Fig. $5 A$, respectively).

\section{Movement representations in parietal cortex and premotor cortex}

As noted above, movements were evoked outside of M1, in PM and parietal areas 1, 2 , and $7 \mathrm{~b}$. In PM, movements of portions of the forelimb were evoked in all cases, although in 1 case (Fig. 1A), there were only 2 stimulation sites in PM. In another case, movements of individual digits alone were evoked in PMv (Fig. 1B); and in the other cases, movements of individual digits and other portions of the forelimb were evoked in PMd (Figs. 1A, $2 A, B)$. In PMd, movements of the shoulder, elbow, and wrist were evoked alone or in combination with other portions of the forelimb. In 3 cases, movements of the hindlimb were evoked in PMd (Figs. 1B, 2); and in 1 of these cases included movements of the toes (Fig. $2 B$ ). Finally, in 2 cases, movements of the ear were also evoked in PMd (Fig. 2); and in 3 cases, movements of different portions of the face and head were evoked in PMv (Figs. 1B, 2).

In anterior parietal areas 1 and 2, the only evoked movements were on portions of the forelimb (Fig. 1 $A, B$ ). However, the majority of the movements evoked were for individual digits. In 1 case, movements of the digits, shoulder, and portions of the face were evoked at a few stimulation sites in area $7 \mathrm{~b}$ (Fig. $1 A$ ).

\section{Movement thresholds in M1}

In $\mathrm{M} 1$, threshold values ranged from 2.5 to $250 \mu \mathrm{A}$ in Case 15-01, 7.5 to $155 \mu \mathrm{A}$ in Case $15-02,15$ to $225 \mu \mathrm{A}$ in Case 15-03, and 35 to $275 \mu \mathrm{A}$ in Case 15-04 (Fig. 10). In 2 cases (Cases 15-01 and 15-04), threshold values in the parietal cortex were relatively higher than in M1 $(p<0.0001)$, but there was no statistically significant difference between thresholds in M1 and PM (Fig. 10). Therefore, differences in threshold value could not be used as a criterion to identify the rostral border of M1. Because our animals were anesthetized, some of the differences in threshold

\section{D1 TO D2} the same as in Figure 6.

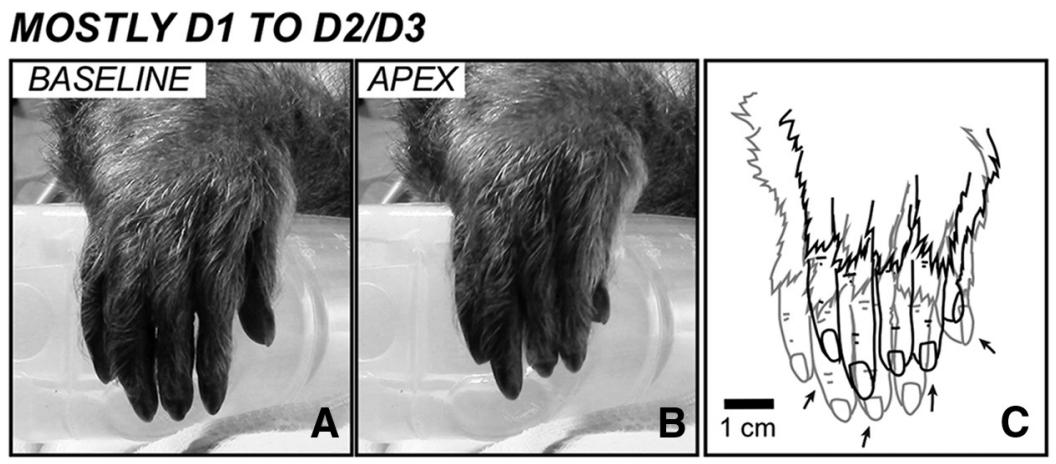

\section{D1 TO ALL FINGERS}
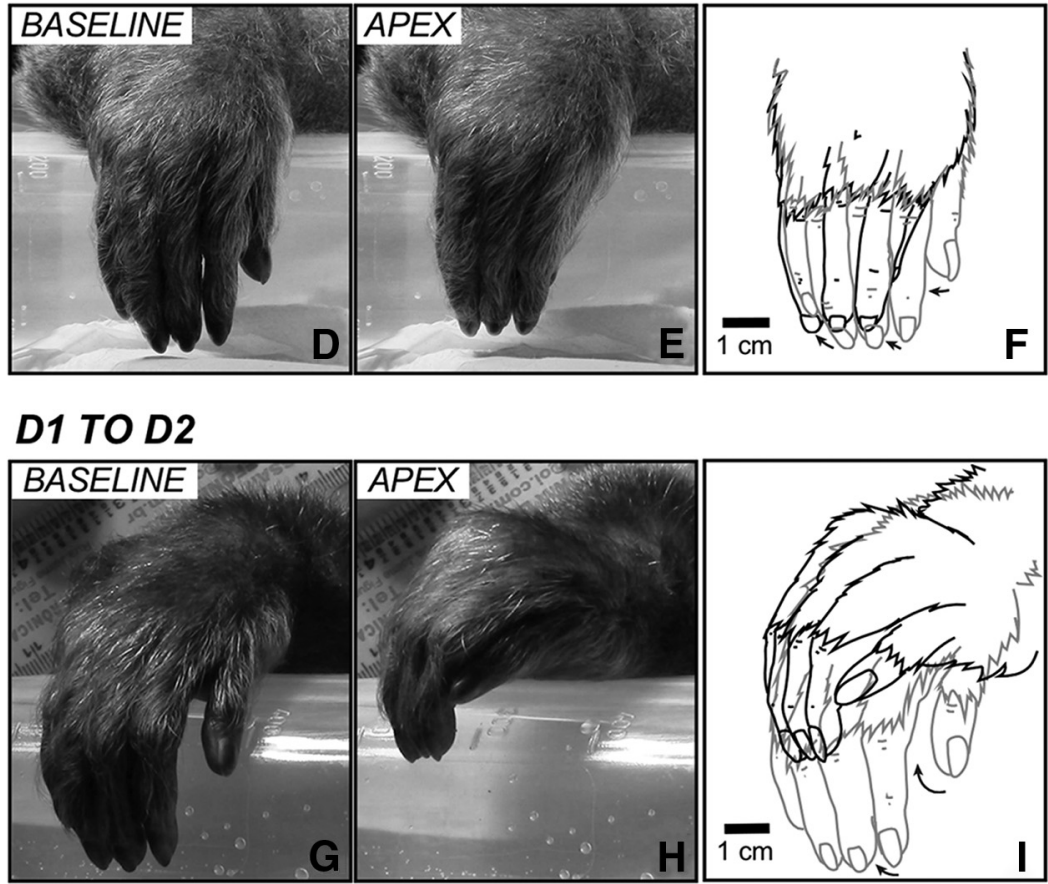

Figure 9. Examples of other types of digit movements. $\boldsymbol{A}-\boldsymbol{C}$, A movement in which there is an abduction/flexion of D1 combined with the flexion of D2 and D3, evoked by LT-ICMS at site 49 of Case 15-01 (see Figure 1-1, available at https://doi.org/10.1523/ JNEUROSCI.0556-19.2019.f1-1). The distal phalange of D1 touches the distal articulation/phalange of D2 and D3. The wrist also flexes dorsally. Other digits also move. $\boldsymbol{D}-\boldsymbol{F}$, An evoked movement in which there is a flexion/abduction of all digits. The tip of D1 列 available at https://doi.org/10.1523/JNEUROSCI.0556-19.2019.f1-1). G-I,D1 moved toward D2 and touched it laterally (the tip of D1 touches the middle phalange of D2), while all other digits abduct and the wrist flexes dorsally and laterally; site 93 of Case 15-01 (see Figure 1-1, available at https://doi.org/10.1523/JNEUROSCI.0556-19.2019.f1-1). Other conventions are

could be due to anesthetic state. M1 threshold levels are consistent with those previously reported for anesthetized macaque monkeys (Baldwin et al., 2018).

\section{Discussion}

Comparisons with behavioral studies in capuchin monkeys In a previous study, manual behavior of capuchin monkeys was videotaped by Costello and Fragaszy (1988), who described criteria to classify power grips and precision grips. In a power grip, all fingers flexed toward the palm, regardless of the position of the thumb relative to the plane of the palm. In a precision grip, the distal phalanx of the thumb touched the middle or distal phalanx of any finger(s), regardless of the movement of the other digits that were not touching the thumb. Spinozzi et al. (2004) subsequently identified 16 variants of the precision grip in capuchin monkeys. 

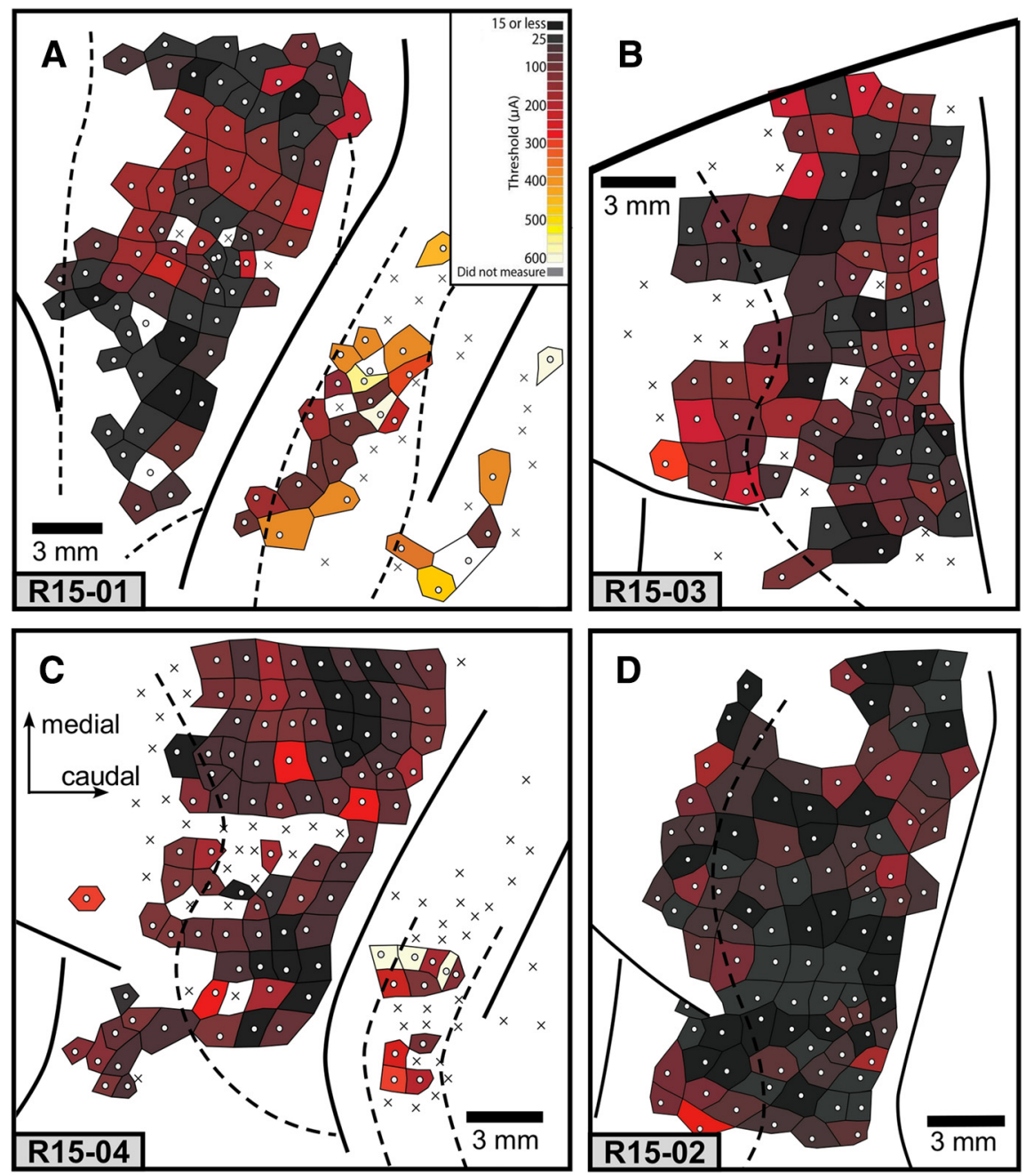

Figure 10. Threshold maps for all cases. A, Case 15-01. B, Case 15-03. C, Case 15-04. D, Case 15-02. Color code in $\boldsymbol{A}$ applies to all figures. Darker tiles represent lower thresholds. Lighter tiles represent higher thresholds. White tiles represent that a threshold was not measured. Other conventions are the same as in Figure 1.

While the movements we evoke do not reflect an intentional manual behavior, we did observe that many ICMS sites from which digit flexions were evoked parallel those of published criteria (Costello and Fragaszy, 1988; Spinozzi et al., 2004) for "precision grip" in which the distal portion of D1 touches any other digit (Figs. 8, 9; Fig. 5 dark purple). However, in some cases, very few sites could be categorized as such (e.g., Case 15-02; Fig. 5D). Thus, the movements we evoked in anesthetized animals were only a subset of those described previously in behaving animals, possibly because we did not survey all of cortex from which movements could be evoked. Still, the fact that we could identify digit movements that resembled specific grip types in previous studies in behaving capuchins, or formed some of the components of complex hand movements, begs the question of what are the basic organizational principles of motor cortex. Obviously, "precision grip-like" movements can be evoked only in primates that have the requisite hand morphology to form a precision grip. Further, it is clear from this study and previous studies in other species that motor cortex organization co-evolved with modifications to forelimb and hand morphology (for review, see Baldwin et al., 2018). We add here the possibility that motor cortex may be constructed during development based on commonly used muscle synergies, many of which may involve social learning (Coelho et al., 2015; Eshchar et al., 2016).

\section{Stimulation of motor cortex} in primates

Over the past century, motor cortex has been explored in a number of primates, including anthropoid apes, using a variety of electrode types and stimulation parameters, in both awake and anesthetized preparations (Leyton and Sherrington, 1917; Woolsey et al., 1952; Kwan et al., 1978; Waters et al., 1990). Despite the differences in the methods used to explore motor cortex, there are several similarities across these studies and across primates. These include a gross topographic organization of movement representations on the precentral gyrus, with a relatively large representation of the hand and forelimb. In some studies, there were large regions of cortex where stimulation evoked finger flexions, similar to those described in the present investigation (e.g., Leyton and Sherrington, 1917; Kwan et al., 1978; Waters et al., 1990).

More recently, LT-ICMS has been used to explore motor and parietal cortex in prosimians (Stepniewska et al., 2005, 2009, 2014), macaques (Griffin et al., 2014; Baldwin et al., 2018), and New World squirrel monkeys and owl monkeys (Gharbawie et al., 2010, 2011a,b; Stepniewska et al., 2014). Results from these studies were similar to those in the present investigation in that a gross topographic organization was observed in motor cortex. Further, movement maps were fractured, with similar body part movements represented at multiple locations. However, there were two important differences between the present study and previous investigations. First, while "power grasping" movements were reported to be evoked in motor cortex of prosimians and New World monkeys, movements that resembled, or could be components of, a precision grip were not evoked in motor cortex, as they were in the capuchins and macaques. Second, in these other species of primates, the cortex that represented the foot and the tail was not explored, or was relatively small compared with this motor representation in capuchin monkeys (see below).

Macaques have an opposable thumb and engage in a variety of dexterous manual behaviors using multiple types of precision grips (Macfarlane and Graziano, 2009). However, previous work that used LT-ICMS to explore motor cortex of macaques described hand movements in a different way (i.e., ICMS-evoked contraction pattern of hand muscles (Park et al., 2001; Overduin et al., 2012; Griffin et al., 2014) or did not provided details of precision-like grips at all (Graziano et al., 2002, 2005; Gharbawie et al., 2011b). An exception was the study of Baldwin et al. (2018), which described the fine details of digit movements evoked by LT-ICMS in motor and parietal cortex. The evoked movements and the maps of M1 in capuchin monkeys strongly resemble the movement representations of macaques in three important ways: (1) M1 was dominated by the forelimb representation; (2) the hand representation in $\mathrm{M} 1$ is located at the level of the superior 
arcuate sulcus; and (3) many of the evoked hand movements, specifically those involving flexion of the digits, were similar to those performed by behaving animals (Macfarlane and Graziano, 2009).

Thus, the organization of the hand representation of capuchin monkeys looks more like that of macaque monkeys (Graziano et al., 2002; Gharbawie et al., 2011b; Rathelot et al., 2017; Baldwin et al., 2018) than that of the more closely related New World monkeys (Gharbawie et al., 2010, 2011a; Stepniewska et al., 2014). Padberg et al. (2007) and Mayer et al. (2016) have observed that the anatomical and functional organization of the parietal cortex of capuchins and macaques are also highly similar. These parallels in the parietal and motor cortices of capuchins and macaques suggest that the sensory-motor circuits that subserve the execution of complex manual behaviors involving complex digit movements, although independently evolved, follow a common principle of organization.

\section{Movements of the prehensile toes and tail}

Many sites involved flexion of all toes (Figs. 5, 7D-I), which could be a movement involved in a variety of behaviors, such as locomotion on tree branches or stabilization of the body with the feet during tool use (Spagnoletti et al., 2011; Mangalam et al., 2018). Curiously, in some cases, ICMS evoked a flexion or abduction of only T1 (Fig. 5), suggesting that motor cortex of capuchin monkeys may also represent the independent movement of toes, which could be an important neural substrate for the execution of relatively precise movements with the toes.

In addition to evoking movements from the toes, we also evoked different types of tail movements, including: (1) the contraction of the base of the tail, and (2) the flexion of the distal portion of the tail. The former type was usually accompanied by trunk and hindlimb movements, and could be related to posture maintenance during the execution of behaviors that require the tail for stabilization (Massaro et al., 2016). The second type of tail movement we observed, flexion of its distal portion, was always isolated from the movement of other body parts, and could be related to grasping behaviors with the tail, such as branchhooking during feeding, foraging, and locomotion (Garber and Rehg, 1999), or pulling food to within reach of the hand (Fulton and Barenne, 1983). This type of tail movement has been observed in ICMS studies of other New World monkeys (i.e., spider monkey and owl monkey) (Fulton and Barenne, 1983; Gould et al., 1986), which also engage in tail prehension, but not in macaques (Fulton and Barenne, 1983; Baldwin et al., 2018), which do not. The relatively small representation of the tail in motor cortex in macaques may be due to the lack of tail prehension in this species or because only a limited extent of cortex in the expected location of the tail representation was explored (Fulton and Barenne, 1983).

In the current study, we evoked four major types of movements of the digits, toes, and tail in M1 that appear to be related to grasping: (1) flexion of all digits toward the palmar surface; (2) flexion of D1 to the distal or middle phalange of other digits; (3) flexion of toes toward the plantar surface; and (4) flexion of the tail tip. Although tail and foot movements may not be directly related to object manipulation, as noted above, they might be relevant to other aspects of behaviors, such as stabilization of the body during use of the hammer and anvil (Mangalam et al., 2018). These data, along with data from other mammals, indicate that motor cortex organization reflects the configuration of peripheral morphology, the biomechanics of different body parts (e.g., an opposable thumb and prehensile tail), and the synergistic use of these body parts. We propose that the execution of complex behavior is generated by the temporally coordinated and combinatorial recruitment of the representations of these muscle synergies in motor cortex.

\section{References}

Asanuma H, Rosén I (1972) Topographical organization of cortical efferent zones projecting to distal forelimb muscles in the monkey. Exp Brain Res $14: 243-256$.

Aversi-Ferreira TA, Maior RS, Carneiro-E-Silva FO, Aversi-Ferreira RA, Tavares MC, Nishijo H, Tomaz C (2011) Comparative anatomical analyses of the forearm muscles of Cebus libidinosus (Rylands et al. 2000): manipulatory behavior and tool use. PLoS One 6:e22165.

Baldwin MK, Cooke DF, Goldring AB, Krubitzer L (2018) Representations of fine digit movements in posterior and anterior parietal cortex revealed using long-train intracortical microstimulation in macaque monkeys. Cereb Cortex 28:4244-4263.

Bortoff GA, Strick PL (1993) Corticospinal terminations in two new-world primates: further evidence that corticomotoneuronal connections provide part of the neural substrate for manual dexterity. J Neurosci 13:5105-5118.

Christel MI, Fragaszy D (2000) Manual function in Cebus apella. digital mobility, preshaping, and endurance in repetitive grasping. Int J Primatol 21:697-719.

Coelho CG, Falótico T, Izar P, Mannu M, Resende BD, Siqueira JO, Ottoni EB (2015) Social learning strategies for nut-cracking by tufted capuchin monkeys (Sapajus spp.). Anim Cogn 18:911-919.

Conrad B, Matsunami K, Meyer-Lohmann J, Wiesendanger M, Brooks VB (1974) Cortical load compensation during voluntary elbow movements. Brain Res 71:507-514.

Costello MB, Fragaszy DM (1988) Prehension in Cebus and Saimiri: I. grip type and hand preference. Am J Primatol 15:235-245.

Côté SL, Hamadjida A, Quessy S, Dancause N (2017) Contrasting modulatory effects from the dorsal and ventral premotor cortex on primary motor cortex outputs. J Neurosci 37:5960-5973.

Dea M, Hamadjida A, Elgbeili G, Quessy S, Dancause N (2016) Different patterns of cortical inputs to subregions of the primary motor cortex hand representation in Cebus apella. Cereb Cortex 26:1747-1761.

Dum RP, Strick PL (2005) Frontal lobe inputs to the digit representations of the motor areas on the lateral surface of the hemisphere. J Neurosci 25: 1375-1386.

Eshchar Y, Izar P, Visalberghi E, Resende B, Fragaszy D (2016) When and where to practice: social influences on the development of nut-cracking in bearded capuchins (Sapajus libidinosus). Anim Cogn 19:605-618.

Fragaszy D, Izar P, Visalberghi E, Ottoni EB, de Oliveira MG (2004) Wild capuchin monkeys (Cebus libidinosus) use anvils and stone pounding tools. Am J Primatol 64:359-366.

Fulton J, Barenne J (1983) The representation of the tail in the motor cortex of primates, with special reference to spider monkeys. J Cel Comp Physiol 2:399-426.

Garber PA, Rehg JA (1999) The ecological role of the prehensile tail in white-faced capuchin (Cebus capucinus). Am J Phys Anthropol 110:325339.

Gharbawie OA, Stepniewska I, Burish MJ, Kaas JH (2010) Thalamocortical connections of functional zones in posterior parietal cortex and frontal cortex motor regions in new world monkeys. Cereb Cortex 20:2391-2410.

Gharbawie OA, Stepniewska I, Kaas JH (2011a) Cortical connections of functional zones in posterior parietal cortex and frontal cortex motor regions in new world monkeys. Cereb Cortex 21:1981-2002.

Gharbawie OA, Stepniewska I, Qi H, Kaas JH (2011b) Multiple parietalfrontal pathways mediate grasping in macaque monkeys. J Neurosci 31: 11660-11677.

Gould HJ 3rd, Cusick CG, Pons TP, Kaas JH (1986) The relationship of corpus callosum connections to electrical stimulation maps of motor, supplementary motor, and the frontal eye fields in owl monkeys. J Comp Neurol 247:297-325.

Graziano MS (2016) Ethological action maps: a paradigm shift for the motor cortex. Trends Cogn Sci 20:121-132.

Graziano MS, Taylor CS, Moore T (2002) Complex movements evoked by microstimulation of precentral cortex. Neuron 34:841-851.

Graziano MS, Aflalo TN, Cooke DF (2005) Arm movements evoked by elec- 
trical stimulation in the motor cortex of monkeys. J Neurophysiol 94:4209-4223.

Griffin DM, Hudson HM, Belhaj-Saiif A, Cheney PD (2014) EMG activation patterns associated with high frequency, long-duration intracortical microstimulation of primary motor cortex. J Neurosci 34:1647-1656.

Gumert MD, Malaivijitnond S (2013) Long-tailed macaques select mass of stone tools according to food type. Philos Trans R Soc Lond B Biol Sci $368: 1-13$.

Gumert MD, Kluck M, Malaivijitnond S (2009) The physical characteristics and usage patterns of stone axe and pounding hammers used by longtailed macaques in the Andaman Sea region of Thailand. Am J Primatol 71:594-608.

Hamadjida A, Dea M, Deffeyes J, Quessy S, Dancause N (2016) Parallel cortical networks formed by modular organization of primary motor cortex outputs. Curr Biol 26:1737-1743.

Heffner RS, Masterton RB (1983) The role of the corticospinal tract in the evolution of human digital dexterity. Brain Behav Evol 23:165-183.

Hoover JE, Strick PL (1999) The organization of cerebellar and basal ganglia outputs to primary motor cortex as revealed by retrograde transneuronal transport of herpes simplex virus type 1. J Neurosci 19:1446-1463.

Jones EG, Coulter JD, Hendry SH (1978) Intracortical connectivity of architectonic fields in the somatic sensory, motor and parietal cortex of monkeys. J Comp Neurol 181:291-347.

Kelly RM, Strick PL (2003) Cerebellar loops with motor cortex and prefrontal cortex of a nonhuman primate. J Neurosci 23:8432-8444.

Kwan HC, MacKay WA, Murphy JT, Wong YC (1978) Spatial organization of precentral cortex in awake primates: II. Motor outputs. J Neurophysiol 41:1120-1131.

Leyton A, Sherrington C (1917) Observations on the excitable cortex of the chimpanzee, orangutan, and gorilla. Exp Physiol 11:135-222.

Lucier GE, Rüegg DD, Wiesendanger M (1975) Responses of neurones in motor cortex and in area 3 a to controlled stretches of forelimb muscles in Cebus monkeys. J Physiol 251:833-853.

Luppino G, Rizzolatti G (2000) The organization of the frontal motor cortex. News Physiol Sci 15:219-224.

Macfarlane NB, Graziano MS (2009) Diversity of grip in Macaca mulatta. Exp Brain Res 197:255-268.

Mangalam M, Rein R, Fragaszy DM (2018) Bearded capuchin monkeys use joint synergies to stabilize the hammer trajectory while cracking nuts in bipedal stance. Proc Biol Sci 285:20181797.

Mannu M, Ottoni EB (2009) The enhanced tool-kit of two groups of wild bearded capuchin monkeys in the Caatinga: tool making, associative use, and secondary tools. Am J Primatol 71:242-251.

Manrique HM, Sabbatini G, Call J, Visalberghi E (2011) Tool choice on the basis of rigidity in capuchin monkeys. Anim Cogn 14:775-786.

Martins AM Jr, Amorim N, Carneiro JC, de Mello Affonso PR, Sampaio I, Schneider H (2015) Alu elements and the phylogeny of capuchin (Cebus and Sapajus) monkeys. Am J Primatol 77:368-375.

Massaro L, Massa F, Simpson K, Fragaszy D, Visalberghi E (2016) The strategic role of the tail in maintaining balance while carrying a load bipedally in wild capuchins (Sapajus libidinosus): a pilot study. Primates 57:231239.

Mayer A, Nascimento-Silva ML, Keher NB, Bittencourt-Navarrete RE, Gattass R, Franca JG (2016) Architectonic mapping of somatosensory areas involved in skilled forelimb movements and tool use. J Comp Neurol 524:1399-1423.

Mayer A, Lewenfus G, Bittencourt-Navarrete RE, Clasca F, da Franca JG (2019) Thalamic inputs to posterior parietal cortical areas involved in skilled forelimb movement and tool use in the capuchin monkey. Cereb Cortex. Advance online publication. Retrieved March 19, 2019. doi: 10.1093/cercor/bhz051.
Moura AC, Lee PC (2004) Capuchin stone tool use in caatinga dry forest. Science 306:1909.

Ohbayashi M, Picard N, Strick PL (2016) Inactivation of the dorsal premotor area disrupts internally generated, but not visually guided, sequential movements. J Neurosci 36:1971-1976.

Overduin SA, d'Avella A, Carmena JM, Bizzi E (2012) Microstimulation activates a handful of muscle synergies. Neuron 76:1071-1077.

Padberg J, Franca JG, Cooke DF, Soares JG, Rosa MG, Fiorani M Jr, Gattass R, Krubitzer L (2007) Parallel evolution of cortical areas involved in skilled hand use. J Neurosci 27:10106-10115.

Park M, Belhaj-Saif A, Gordon M, Cheney PD (2001) Consistent features in the forelimb representation of primary motor cortex in rhesus macaques. J Neurosci 21:2784-2792.

Quessy S, Côté SL, Hamadjida A, Deffeyes J, Dancause N (2016) Modulatory effects of the ipsi and contralateral ventral premotor cortex (PMv) on the primary motor cortex (M1) outputs to intrinsic hand and forearm muscles in Cebus apella. Cereb Cortex 26:3905-3920.

Rathelot JA, Dum RP, Strick PL (2017) Posterior parietal cortex contains a command apparatus for hand movements. Proc Natl Acad Sci U S A 114:4255-4260.

Rosén I, Asanuma H (1972) Peripheral afferent inputs to the forelimb area of the monkey motor cortex: input-output relations. Exp Brain Res 14:257-273.

Schindelin J, Arganda-Carreras I, Frise E, Kaynig V, Longair M, Pietzsch T, Preibisch S, Rueden C, Saalfeld S, Schmid B, Tinevez JY, White DJ, Hartenstein V, Eliceiri K, Tomancak P, Cardona A (2012) Fiji: an open source platform for biological image analysis. Nat Methods 9:676-682.

Spagnoletti N, Visalberghi E, Ottoni E, Izar P, Fragaszy D (2011) Stone tool use by adult wild bearded capuchin monkeys (Cebus libidinosus): frequency, efficiency and tool selectivity. J Hum Evol 61:97-107.

Spinozzi G, Truppa V, Laganà T (2004) Grasping behavior in tufted capuchin monkeys (Cebus apella): grip types and manual laterality for picking up a small food item. Am J Phys Anthropol 125:30-41.

Spinozzi G, Laganà T, Truppa V (2007) Hand use by tufted capuchins (Cebus apella) to extract a small food item from a tube: digit movements, hand preference, and performance. Am J Primatol 69:336-352.

Stepniewska I, Fang PC, Kaas JH (2005) Microstimulation reveals specialized subregions for different complex movements in posterior parietal cortex of prosimian galagos. Proc Natl Acad Sci U S A 102:4878-4883.

Stepniewska I, Fang PC, Kaas JH (2009) Organization of the posterior parietal cortex in galagos: I. Functional zones identified by microstimulation. J Comp Neurol 517:765-782.

Stepniewska I, Gharbawie OA, Burish MJ, Kaas JH (2014) Effects of muscimol inactivations of functional domains in motor, premotor, and posterior parietal cortex on complex movements evoked by electrical stimulation. J Neurophysiol 111:1100-1119.

Visalberghi E, Fragaszy D, Ottoni E, Izar P, de Oliveira MG, Andrade FR (2007) Characteristics of hammer stones and anvils used by wild bearded capuchin monkeys (Cebus libidinosus) to crack open palm nuts. Am J Phys Anthropol 132:426-444.

Visalberghi E, Addessi E, Truppa V, Spagnoletti N, Ottoni E, Izar P, Fragaszy D (2009) Selection of effective stone tools by wild bearded capuchin monkeys. Curr Biol 19:213-217.

Vogt C, Vogt O (1919) Allgemeine ergebnisse unserer hirnforschung. J Psychol Neurol 25:279-462.

Waters RS, Samulack DD, Dykes RW, McKinley PA (1990) Topographic organization of baboon primary motor cortex: face, hand, forelimb, and shoulder representation. Somatosens Mot Res 7:485-514.

Woolsey CN, Settlage PH, Meyer DR, Sencer W, Pinto Hamuy T, Travis AM (1952) Patterns of localization in precentral and "supplementary" motor areas and their relation to the concept of premotor area. Res Publ Assoc Res Nerv Ment Dis 30:238-264. 